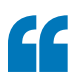

We discovered how targeting a metabolic pathway renders $V H L$ deficient cancer cells sensitive to DNA repair mechanisms
$\Rightarrow$ KIDNEY CANCER

\section{Targeting metabolism in RCC}

Loss of VHL and elevated expression of HIF transcription factors leads to reprogramming of tumour cell metabolism, enabling renal cell carcinoma (RCC) cells to use carbons from glutamine-derived aspartate to drive pyrimidine biosynthesis. Now, researchers show that inhibition of glutamine availability decreases aspartate availability, leading to growth inhibition of RCC cells. Moreover, glutaminase 1 (GLS1) inhibition increases oxidative stress, leading to DNA replication stress and growth arrest. "These findings led us to combine a GLS1 inhibitor with an inhibitor of DNA repair enzymes - in this case a poly(ADPribose) polymerase (PARP) inhibitor - to achieve therapeutic synergism," explains Othon Iliopoulos. "In other words, we discovered how targeting a metabolic pathway renders $V H L$-deficient cancer cells sensitive to DNA repair mechanisms."

Iliopoulos explains that the goal of their project was to understand the mechanisms responsible for growth suppression of renal cancer cells following GLS1 inhibitor treatment, and to use this knowledge to devise a rational therapy to target cancers that express high levels of HIF. They showed that in $V H L$-deficient RCC cells, which express high levels of HIF, glutamine carbons feed de novo pyrimidine synthesis and sustain nucleotide levels required for DNA replication. "Thus, renal cancer cells treated with a GLS1 inhibitor fail to synthesize enough nucleotides to support their DNA replication and show signs of pronounced DNA replication stress and DNA damage," says Iliopoulos. GLS1 inhibition also suppressed glutathione synthesis from glutamine carbons, leading to increased intracellular levels of reactive oxygen species, which induced DNA replication stress independently of suppressed pyrimidine synthesis. Importantly, combination treatment with a PARP inhibitor and a GLS1 inhibitor synergistically inhibited growth of $V H L$-deficient RCC cells in mice.

Iliopoulos hopes to take their findings directly to a clinical trial. "We now plan to treat patients with renal and other HIF-expressing cancers with this novel combination of GLS1 and PARP inhibitors," he explains.

Susan. J. Allison

ORIGINAL ARTICLE Okazaki, A. et al. Glutaminase and poly(ADP-ribose) polymerase inhibitors suppress pyrimidine synthesis and VHL-deficient renal cancers. J. Clin. Invest. http://dx.doi.org/10.1172/JC187800 (2017) 